\title{
COMPOSICIÓN Y ESTRUCTURA AVIAR EN DOS PARCHES DE BOSQUE SECO EN EL VALLE DEL CAUCA
}

\author{
Juliana Tamayo-Quintero ${ }^{1}$, Lorena Cruz-Bernate ${ }^{2}$
}

\section{Resumen}

El bosque seco es uno de los ecosistemas más degradados, fragmentados y menos conocidos, destruidos para implementar ciudades, cría ganadera y cultivos, quedando solo pequeños parches considerados importantes reservorios de diversidad biológica. Dado la ausencia de información sobre avifauna en los parches de bosque seco aún presentes en el Valle del Cauca y su papel en el mantenimiento de las poblaciones de aves, el objetivo de este estudio fue determinar y comparar la estructura y composición de la avifauna de los parches de bosque Las Chatas y Colindres y compararla en contexto con otras áreas arbóreas de bosque seco de la región. En cada bosque se realizaron tres días de muestreo por mes, entre julio y diciembre de 2012. Se emplearon recorridos de avistamiento por senderos, puntos de conteo, e instalación de redes para el monitoreo de las aves. Se registraron en total 76 especies de aves, pertenecientes a 28 familias y 14 órdenes. Cinco especies migratorias, nueve medianamente amenazadas y dos endémicas regionales fueron detectadas. Aunque la riqueza de especies y grupos tróficos fue similar ( $p>0,05)$, el bosque Colindres presentó una ligera mayor diversidad $\left(\mathrm{H}^{\prime}=3,36\right.$ y 2,95 en Las Chatas), sustentada en la mayor riqueza vegetal y calidad de la matriz. Aunque ambos bosques son de igual tamaño, sólo compartieron el $46 \%$ de la avifauna, lo que sugiere complementariedad en riqueza de especies para permitir la sostenibilidad de la diversidad. Es importante implementar estrategias de conservación que permitan restaurar y recuperar estos parches de bosque, que son al parecer un refugio importante para las aves de la región.

Palabras clave: abundancia, Chatas, Colindres, Colombia, diversidad de aves, matriz, riqueza.

\section{BIRD COMPOSITION AND STRUCTURE ON TWO PATCHES OF DRY FOREST IN THE VALLEY OF CAUCA}

\begin{abstract}
The dry forest is one of the most degraded, fragmented and less known ecosystems, it is being transformed to implement cities, livestock and crops, leaving only small forest patches which are considered important reservoirs of biodiversity. Given the absence of birdlife information on patches of dry forest still present in the Valle del Cauca and their role in maintenance of the bird populations, the objective of this study was to determine and compare the structure and composition of the avifauna in the forest patches the Chatas and Colindres, and to compare their avifauna with other dry forest areas of the region. In each forest, three sampling

\footnotetext{
${ }^{*}$ FR: 10-III-2014, FA: 20-II-2015

${ }^{1}$ Departamento de Biología, Universidad del Valle, Calle 13 No. 100-00, A. A. 25360,Cali, Colombia. E-mail: juliana.tamayo@correounivalle.edu.co

${ }^{2}$ Departamento de Biología, Universidad del Valle, Calle 13 No. 100-00, A. A. 25360,Cali, Colombia. E-mail: lorena.cruz@correounivalle.edu.co
} 
days per month were performed from July until December in 2012. Counting points and bird captures were done to monitor individuals. Seventy-six species of birds, belonging to twenty-eight families and fourteen orders were recorded. Five migratory species, nine moderately endangered and two regional endemic species were found. Although species richness and trophic groups was similar ( $p>0,05)$, the Colindres forest had a greater diversity ( $\mathrm{H}$ '=3.36 and 2.95 for "Las Chatas"), supported for high plant richness and quality of its landscape matrix. Although both forests are of similar size, they shared $46 \%$ of birdspecies, being they complementary for conservation of bird populations. It is important to implement a monitoring and conservation strategies that allow the restoration or recovery of these forest patches, which are important refuges of bird species.

Key words: abundance, Chatas, Colindres, Colombia, bird diversity, matrix, richness.

\section{INTRODUCCIÓN}

El bosque seco tropical es uno de los ecosistemas más importantes en el mantenimiento y conservación de poblaciones de aves, tanto locales como migratorias transcontinentales en el Neotrópico (KRICHER, 2010). A pesar de ello, es considerado actualmente como uno de los ecosistemas más degradados, fragmentados y menos investigados en el mundo (MURPHY \& LUGO, 1986; JANZEN, 1988; GARCÍA-OLIVA et al. 1994; QUESADA \& STONER, 2003). Una de las causas de su estado actual es que, debido a lo atractivo que ha resultado para el hombre las condiciones climáticas y edáficas de esta zona de vida, éste ha ejercido una fuerte presión negativa durante décadas, donde se ha implementado el fuego como mecanismo de destrucción rápida para abrir paso al uso del suelo con fines agropecuarios y urbanísticos (SALAZAR-RAMÍREZ et al. 2002). Actualmente estos bosques representan solo el $42 \%$ de los bosques tropicales y subtropicales del mundo, lo que corresponde al 0,1\% de su cobertura original (MURPHY \& LUGO, 1986; JANZEN, 1988). Las actividades antropogénicas, han sido una de las fuerzas con mayor efecto en el moldeamiento del paisaje a mosaicos de parches naturales (LUQUE et al. 1994).

Cambios estructurales en el hábitat tienen efecto en las dinámicas poblacionales al interior de las áreas boscosas fragmentadas, que incluyen desde reducción del número de individuos hasta pérdidas irrecuperables de la biodiversidad (KATTAN \& ÁLVAREZ-LÓPEZ, 1996). Hay evidencia que la biodiversidad está influenciada por el tamaño del hábitat disponible y la heterogeneidad vegetal (FREEMARK \& MERRIAN, 1986; KATTAN \& ÁLVAREZ-LÓPEZ, 1996; PERFECTO et al. 2009), donde parches de mayor área y heterogeneidad, presentan un mayor número y proporción de especies propias de interior de bosque que aquellos pequeños; y la oferta de recursos, también es mayor, propiciando la existencia de diferentes grupos tróficos (GALLI et al. 1976; WILLIS, 1979; FREEMARK \& MERRIAN, 1986; BERGET, 2006). Adicionalmente, las especies que requieren grandes áreas y doseles maduros para la búsqueda de alimento, que se encuentren en su límite altitudinal de distribución y requieran sotobosques no intervenidos, son consideradas bioindicadoras ambientales, ya que son las más vulnerables (RUDNICKY \& HUNTER, 1993; KATTAN et al. 1994; KATTAN \& ÁLVAREZ-LÓPEZ, 1996). 
En regiones fragmentadas, la calidad de la matriz también puede ser un factor determinante en la diversidad de especies y gremios que persisten a escala del paisaje (PERFECTO et al. 2009). Los tipos de hábitats que existen en la matriz pueden ser muy importantes para el desplazamiento de organismos dentro del hábitat y determinan la probabilidad que ciertas especies sean viables (KATTAN \& ÁLVAREZLÓPEZ, 1996; CÁRDENAS, 1998; PERFECTOet al. 2009).El bosque seco en Colombia, que se presenta en fragmentos y parches rodeados de diferentes tipos de matrices, está ubicado en las regiones de la llanura Caribe y valles interandinos de los ríos Magdalena y Cauca entre 0 y 1000 m de altitud, en los Departamentos del Cauca, Valle del Cauca, Tolima, Huila, Cundinamarca, Antioquía, Sucre, Bolívar, Cesar, Magdalena, Atlántico y sur de la Guajira (CHAVES \& ARANGO, 1998). En el Valle del Cauca, se ha reportado una disminución de la fauna asociada a estos bosques, debido probablemente a los efectos negativos del aislamiento y la fragmentación de los remanentes boscosos (CHAVES \& ARANGO, 1998). ÁLVAREZ et al. (1991), reportaron una muy baja densidad poblacional y/o extinción local de 40 especies de aves para este departamento.

Los parches de bosque seco aún existentes en el Valle del Cauca, se degradan a una velocidad mayor que el conocimiento que se produce con la investigación científica sobre ellos (CHAVES \& ARANGO, 1998; CORPORACIÓN AUTÓNOMA REGIONAL DEL VALLE DEL CAUCA-CVC, 1990; AMBRECHT Y ULLOA-CHACÓN, 1999; SALAZARRAMÍREZet al. 2002). Se conoce un poco sobre la diversidad de la avifauna presente en el Enclave seco del Río Amaime (CÁRDENAS et al. 2013), El Hatico (CÁRDENAS, 1998), Formosa: una finca ganadera en el norte del Valle del Cauca, Club Farallones de Cali, el CIAT, parques boscosos de la ciudad de Cali, La Buitrera, La Universidad del Valle (NARANJO, 1992; NARANJO \& ESTELA, 1999; SEDANO, 2000; ANGARITA, 2002; RIVERA-GUTIÉRREZ, 2006; MUÑOZ et al. 2007) y del bosque El Castillo cerca de Colindres (OREJUELA et al. 1979; OREJUELA, 1979), pero se desconoce la avifauna que se encuentra en los parches de Colindres y Las Chatas. El único trabajo con un esfuerzo acumulado de varios años que contempla este tipo de hábitat en el Valle del río Cauca, es el de ÁLVAREZ-LÓPEZ (1999) sobre la avifauna de la Reserva Natural Laguna de Sonso; en él se registran las aves acuáticas y las especies asociadas a hábitats menos húmedos, con un total de 144 especies. Estudios de éste tipo y análisis detallados sobre la estructura y composición de comunidades de aves, permiten entender los parámetros que rigen las relaciones tróficas y ambientales para el mantenimiento de las poblaciones de aves a nivel de paisaje (ORIANS, 1969; VILLAREAL et al. 2004; CÁRDENAS, 1998). El conocimiento completo y comparativo de la avifauna de cualquier región, es de suma importancia tanto para la toma de decisiones en términos de manejo y conservación, como para las actividades de esparcimiento de tipo educativo y de concientización (ANGARITA, 2002). Por lo tanto es necesario la implementación de monitoreos de poblaciones a largo plazo, que permitan incrementar el conocimiento de la dinámica poblacional y cómo las especies responden a los cambios ambientales a través el tiempo.

Debido a la falta de información actual sobre riqueza de especies aviarias de los parches de bosque seco en el Valle del Cauca y a la situación crítica por la que atraviesan estas zonas boscosas por la notable reducción de área, Las Chatas y Colindres se convierten en sitios importantes para el estudio de la riqueza, abundancia y composición de la avifauna residente y migratoria. El objetivo de este estudio fue estimar y comparar la composición y estructura de la avifauna de estos dos parches de bosque seco tropical ubicados en matrices de heterogeneidad diferente en el Departamento del Valle del Cauca, Colombia. 


\section{MÉTODOS}

Áreas de estudio:El bosque Las Chatas se encuentra ubicado en el centro del Departamento del Valle del Cauca, Municipio de Guadalajara de Buga a $03^{\circ} 51$ '20,8”N-76²0'5,35” W, 950 msnm, en la zona de amortiguamiento del AICA -Laguna de Sonso. Tiene una extensión de 10 ha y una matriz conformada de monocultivo de caña de azúcar, terreno arado permanentemente, sin cobertura vegetal en su mayoría y potrero ganadero (Tamayo-Quintero Obs. pers).

El bosque Colindres se encuentra en el sur del Departamento del Valle del Cauca,

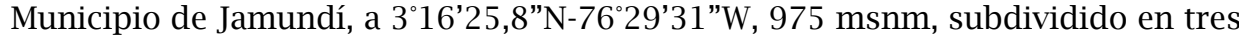
parches. Tiene una extensión de 12,8 ha, y una matriz conformada principalmente por potrero arbolado con mantecos (Laetia americana) y caña de azúcar en el borde sur y oriental. Ambos bosques, según el sistema de clasificación de Holdridge (ESPINAL, 1967), son considerados Bosque Seco Tropical (bs-T), con temperatura y precipitación promedio anual de $24^{\circ} \mathrm{C}$ y $1350 \mathrm{~mm}$, respectivamente (CHAVES \& ARANGO, 1998). La lluvia se distribuye en dos temporadas, de marzo a mayo y de septiembre a noviembre. La humedad relativa alcanza un promedio de $70-75 \%$. Las características de la estructura de la vegetación arbórea de Las Chatas, muestra que algunos individuos alcanzan un DAP hasta de $46 \mathrm{~cm}$, pero el 65\% se encuentra en clases diamétricas más bajas, indicando que es un bosque de árboles jóvenes que se encuentran en proceso de colonización del dosel, con una gran cantidad de claros. En el bosque Colindres, el 70\% de los individuos registran un DAP máximo de $23 \mathrm{~cm}$; mientras que, el 0,5\% tienen un DAP superior de $90 \mathrm{~cm}$. Sólo el 21\% de los individuos se encuentran establecidos y en proceso de ocupación del dosel (CÁRDENAS et al. 2012).

Métodos de campo. El trabajo se llevó a cabo entre julio y diciembre de 2012. En cada bosque se hicieron visitas mensuales de tres días de muestreo en las cuáles se emplearon tres métodos para la determinación de la avifauna: observación por senderos, puntos de conteo y captura de individuos con redes de niebla. El método de senderos consistió en registrar todas las aves presentes a lado y lado del observador a medida que se caminaba a una velocidad constante por el trayecto establecido (VILLAREAL et al. 2004), éstos tuvieron un ancho de $25 \mathrm{~m}$ por $980 \mathrm{~m}$ de largo aproximadamente y se recorrieron a una velocidad de $0.245 \mathrm{~km} / \mathrm{h}$. El método de puntos de conteo consistió en la observación y conteo de todas las aves presentes alrededor del observador el cual permaneció en sitios fijos. El radio de cada punto fijo fue de $25 \mathrm{~m}$ con una separación mínima de $200 \mathrm{~m}$ entre ellos (RALPH et al. 1995). Se ubicaron diez puntos de conteo dentro de cada bosque y dos en la matriz, con dedicación de diez minutos continuos de observación en cada uno y cinco min al desplazamiento entre ellos, los puntos fijos se ubicaron en coincidencia con los senderos de observación. Se recorrió todo el bosque dos veces por día entre 06:00 y 10:00 h y entre 14:00 y 18:00 h. Durante el avistamiento, cada individuo se identificó hasta especie, se registró su estructura social (solitario, pareja, grupo mixto, bandada, etc.), sexo en caso de presentarse dimorfismo sexual $\mathrm{y}$ actividad en general.

Para obtener información sobre especies de difícil detección, se cubrieron 60 m de longitud con seis redes de niebla ( $10 \mathrm{~m}$ largo X $2.5 \mathrm{~m}$ ancho), ojo de malla de 30 $\mathrm{mm}$, tres a $3 \mathrm{~m}$ de altura y tres a $5 \mathrm{~m}$ de altura, abiertas entre las 6:00 y 17:30 h. De cada individuo capturado se registraron las medidas morfométricas de peso, 
longitud de ala, rectrices, tarso-metatarso, largo y ancho del pico empleando una balanza (Pesola) $\pm 0,3$ de precisión y un calibrador digital $( \pm 0,01 \mathrm{~mm})$. Para la identificación hasta especie se utilizaron la Guía de aves de Colombia y la Guía de Campo de Aves de Norteamérica (HILTY \& BROWN, 2001; KAUFFMAN, 2005), con la actualización taxonómica a 2014 para Sur América, generada por el comité de clasificación de la Unión Americana de Ornitólogos, AOU (REMSEN et al. 2014).

Para determinar el área, perímetro y forma de cada parche de bosque, se registró con un GPS las coordenadas de todo el borde; también se utilizó el programa QGIS Valmiera 2.2 (2014) y fotografías digitales donadas por la Corporación Autónoma Regional del Valle del Cauca-CVC (2014). Los datos de la composición vegetal de los parches de bosque y sus matrices, fueron tomados como información secundaria del proyecto "Monitoreo de la diversidad de plantas presentes en dos parches de Bs-T Colindres y Las Chatas", realizado dentro del convenio No. 0008 entre la Universidad del Valle y la Corporación Autónoma Regional del Valle del CaucaCVC. La caracterización vegetal, siguió el protocolo propuesto por LOZANO et al. (2009), ajustado para hábitats fragmentados. La información sobre precipitación, temperatura media y humedad relativa, fueron suministrados por el Centro de Investigación de la Caña de Azúcar de Colombia, (CENICAÑA, 2014).

Análisis estadísticos: Para evaluar la representatividad del muestreo de aves, se siguió el método de VILLAREAL et al. (2004): cálculo del estimador ACE como medida de la riqueza esperada y curvas de acumulación de especies con el programa EstimateS 7.0 (COLWELL, 2005). La abundancia relativa se determinó mediante la frecuencia de individuos de cada especie registrada durante el muestreo, se calculó como $\mathrm{Pi}=(\mathrm{nsi} / \Sigma \mathrm{n}) \times 100$; donde $\mathrm{Pi}$, representa la abundancia relativa de la especie i, n si el número de individuos de la especie i y $\Sigma$ n el número total de individuos (PETTINGIL, 1969). Se establecieron cuatro categorías de abundancia: rara (0,08$1,9)$, poco común $(2,0-4,9)$, común $(5,0-9,9)$ y abundante $(10-24,5)$.

Para estimar la diversidad de especies se empleó el índice de Shannon (H') (MAGURRAN, 1988), utilizando el programa PAST 2.17 (HAMMERet al. 2001), y su resultado se transformó en "número efectivo" expresado como $\mathrm{D}=\exp \left(\mathrm{H}^{\prime}\right)$ (JOST, 2006), el cual mide la diversidad que tendría una comunidad integrada por i especies igualmente comunes. Las especies se clasificaron, según el principal tipo de alimento consumido, en uno de los siguientes grupos tróficos AO: acuático-omnívoro, C: carroña, F: frutas, FS: frutas y semillas, IP: insectos e invertebrados pequeños, IP-F: insectos, invertebrados pequeños y frutas, IP-IV: insectos, invertebrados pequeños, invertebrados grandes y vertebrados muy pequeños, N-IP: néctar, insectos e invertebrados pequeños, S: semillas, SF: semillas y frutas, V: vertebrados grandes y IV: invertebrados grandes y vertebrados muy pequeños (STILES \& ROSELLI, 1998). Se empleó la prueba Chi-cuadrado de dos muestras independientes (ZAR, 1999), para evaluar si existían diferencias significativas en la riqueza, abundancia y composición de aves entre los bosques Las Chatas y Colindres. Los mismos parámetros se analizaron entre las matrices de los dos bosques y se exploró la relación de ellos con variables ambientales de precipitación, temperatura y humedad relativa. Se usó el índice de correlación de Spearman (ZAR, 1999), para determinar si existe una interrelación entre riqueza de especies de aves y riqueza vegetal de los sitios estudiados. Adicionalmente, se analizó la similitud de especies que presentaban los parches de bosques estudiados con otras áreas de vegetación de bosque seco conocidas en el Valle del Cauca, a través el índice de similitud de Jaccard (ZAR, 
1999). Finalmente, se evaluaron los dos métodos de muestreo de observación por recorrido y puntos de conteo para conocer cuál de ellos fue más informativo en los bosques Colindres y Las Chatas.

\section{RESULTADOS}

Un total de 76 especies de aves fueron registradas, pertenecientes a 28 familias (Anexo 1). Tyrannidae, Thraupidae y Ardeidae fueron las que presentaron mayor número de especies tanto en los bosques como en las matrices (Fig. 1). Las curvas de acumulación de especies sugieren un esfuerzo de muestreo suficiente para el análisis de la avifauna (Fig. 2).
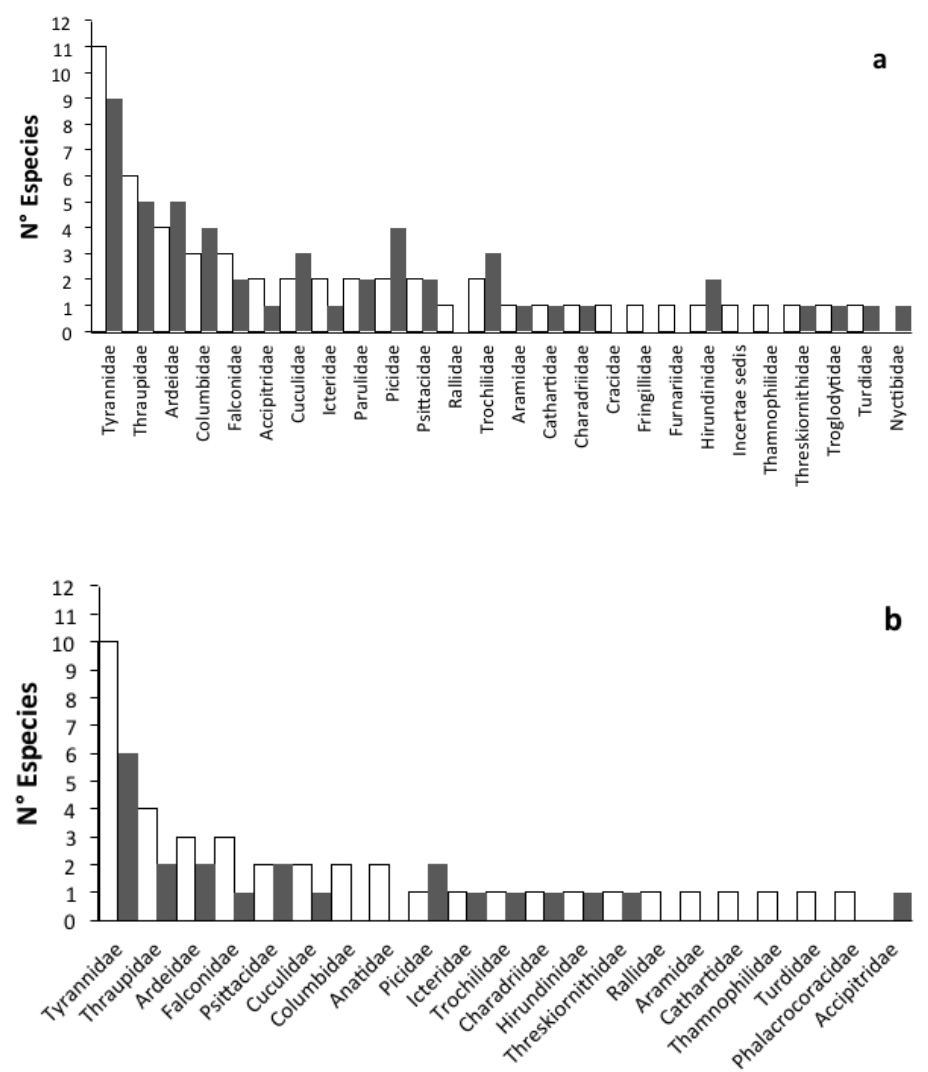

Familias

$\square$ Colindres $\quad$ Chatas

Figura 1. Número de especies de aves por familia en los parches de bosque seco Colindres y Las Chatas (a), y sus respectivas matrices (b) en el Valle del Cauca, suroccidente de Colombia. 

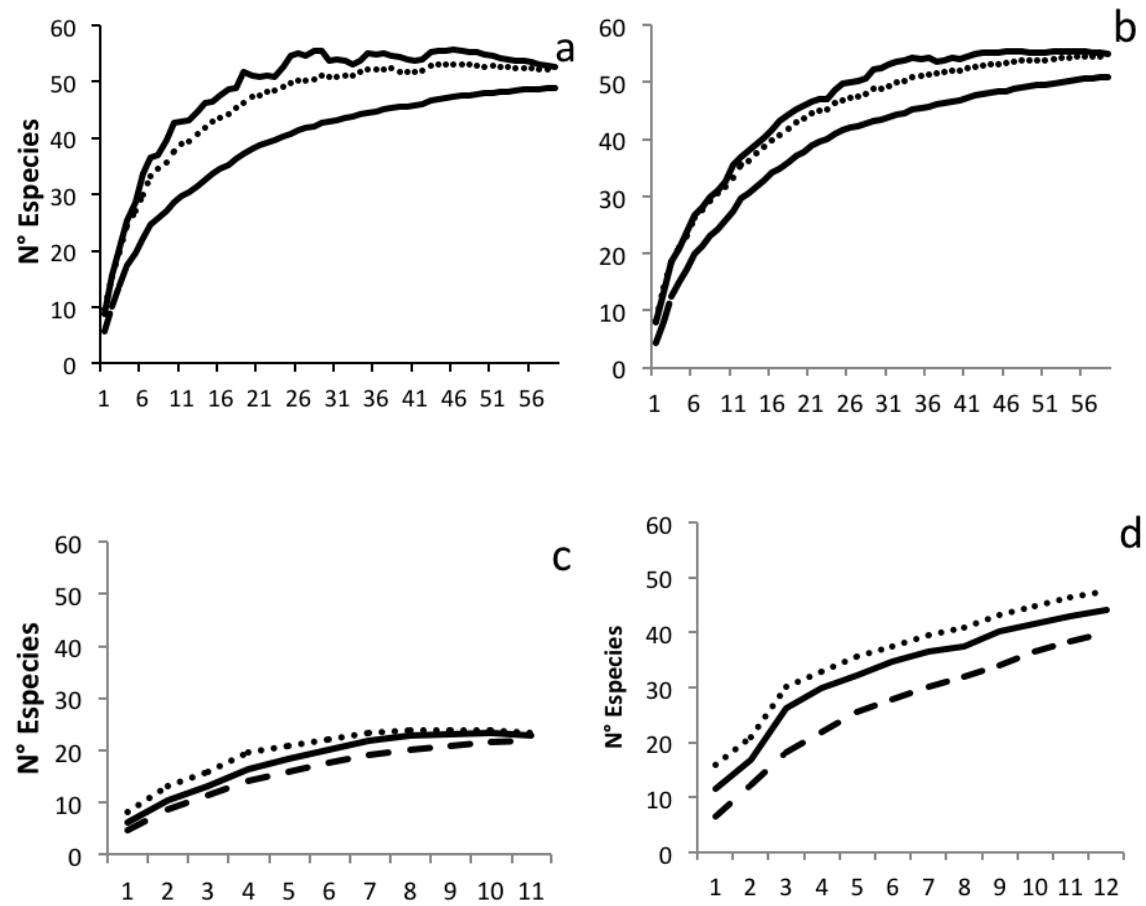

C

Muestras

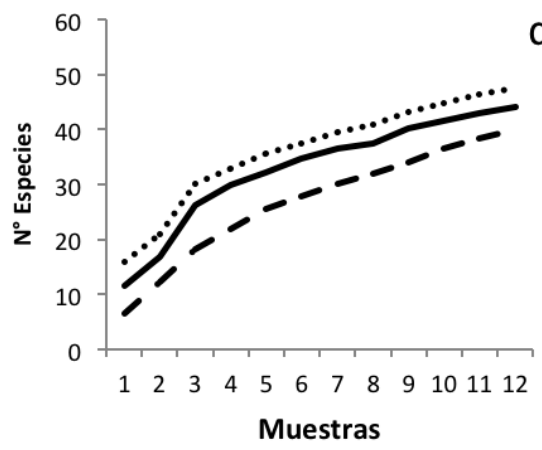

- - Observados $\cdots \cdot \cdot$ ACE $\longrightarrow$ Chao 1

Figura 2. Curvas de acumulación de especies de aves observadas y esperadas en los parches de bosque seco Las Chatas (a), Colindres (b), Matriz Las Chatas (c) y Matriz Colindres (d) con esfuerzo de muestreo del $94,1 \%, 93,5 \%, 94,4 \%$ y $84,1 \%$, respectivamente, en el Valle del Cauca, suroccidente de Colombia.

El número de especies entre los bosques fue similar $\left(\chi_{1: 0,05}^{2}=0,15 ; p=0,69\right)$. En Las Chatas se registraron 50 especies de aves, distribuidas en 20 familias, de éstas especies cuatro fueron migratorias: Catharus ustulatus, Myiarchus crinitus, Hirundo rustica y Setophaga petechia. En Colindres se registraron 54 especies de aves, distribuidas en 25 familias, con una especie migratoria, Geothlypis philadelphia. La riqueza de las matrices de los bosques presentó diferencias significativas $\left(\chi_{1: 0,05}^{2}=5,23 ; p=0,02\right)$. En la matriz de Las Chatas se registraron 22 especies de 13 familias y en la matriz de Colindres 40 especies de 20 familias. Se registró la migratoria Anas discors en la matriz de Colindres. Algunas especies se registraron exclusivamente en la matriz de cada bosque, Phaeomyias murina para la matriz de las Chatas y Anas discors, Dendrocygna bicolor, Phalacrocorax brasilianus, Pyrocephalus rubinus y Porphyrio martinicus para la matriz de Colindres.Las especies observadas al interior delos bosques que no se detectaron en la matriz fueron Myiarchus apicalis para Colindres y Piaya cayana, Nyctibius griseus y Leptotila plumbeiceps para Las Chatas. El análisis comparativo de las especies presentes entre el borde de bosque y su interior, muestra una similitud de 98,1\% en Colindres y 94\% en Las Chatas (índice Jaccard); lo que sugiere que no existe un interior propiamente dicho. 
Se registraron especies medianamente amenazadas a nivel regional (CASTILLO \& GONZÁLEZ, 2007) tanto en los bosques como en las matrices: Ardea cocoi, Dendrocygna bicolor, Anas discors, Rosthramus sociabilis, Falco femoralis, Ortalis columbiana, Aramides cajaneus, Pionus menstruus y Crotophaga major, así como también, especies endémicas regionales (Stotzet al. 1996) dentro de cada bosque como Myiarchus apicalis en el parche de Colindres y Picumnus granadensis en Las Chatas.

Se observó comportamiento reproductivo de algunas especies. Por ejemplo, en Las Chatas, Falco femoralis construyó nido en octubre y Milvago chimachima copuló en julio. En Colindres se observó a Columbina talpacoti en cortejo durante septiembre, Falco sparverius alimentó juveniles en julio, Pitangus sulphuratus y Zenaida auriculata construyeron nidos en noviembre y julio, respectivamente, y adultos de Ortalis columbiana fueron observados en compañía de varios juveniles en noviembre.

El número de especies por familia entre los bosques Colindres y Las Chatas fue similar $\left(\chi_{25 ; 0,05}^{2}=10,99 ; p=0,99\right)$, al igual que en las matrices $\left(\chi_{20 ; 0,05}^{2}=10,16 ; p=0,96\right)$. La riqueza de especies fue constante durante el tiempo de muestreo en cada bosque y entre ellos (Colindres $\chi_{4 ; 0,05}^{2}=6,72 ; \mathrm{p}=0,15$, Chatas $\chi_{4 ; 0,05}^{2}=1,35 ; \mathrm{p}=0,85$; entre bosques $\chi_{4 ; 0,05}^{2}=2,39 ; p=0,66$ ) (Fig. 3), al igual que entre sus matrices $\left(\chi_{4 ; 0,05}^{2}=8,48 ; p=0,075\right.$ ). Sin embargo, cuando se examinó la riqueza en las matrices de manera individual, se encuentra que en la matriz de Colindres hubo variación significativa con el tiempo (Colindres $\chi_{4 ; 0,05}^{2}=22,17 ; p=0,001$ y Chatas $\chi_{4 ; 0,05}^{2}=2,48 ; p=0,65$ ) (Fig. 3).

La variación temporal de la abundancia difirió significativamente en cada bosque y entre ellos (Colindres $\chi_{4 ; 0,05}^{2}=98,67 ; \mathrm{p}=0,001$, Chatas $\chi_{4 ; 0,05}^{2}=1077,62 ; \mathrm{p}=0,001 \mathrm{y}$ entre bosques $\chi_{4 ; 0,05}^{2}=49,4 ; \mathrm{p}=0,001$ ) (Fig. 3), siendo Las Chatas el sitio con mayor número de individuos. Entre las matrices, no hubo diferencias en la variación temporal de la abundancia $\left(\chi_{4 ; 0,05}^{2}=8,48 ; p=0,075\right)$; sin embargo, al examinarlas de manera individual, la abundancia si varió con el tiempo en la matriz de Colindres (Colindres $\chi_{4 ; 0,05}^{2}=115 ; \mathrm{p}=0,001$ y Chatas $\chi_{4 ; 0,05}^{2}=1,93 ; \mathrm{p}=0,75$ ) (Fig. 3).

La temperatura y humedad relativa en ambos sitios permanecieron constantes (Colindres: Temperatura promedio $=23,6^{\circ} \mathrm{C}$, ds $=0$, Tmáx $=23,9^{\circ} \mathrm{C}$, Tmín $=23,1^{\circ} \mathrm{C}, \mathrm{n}=6$; Humedad relativa promedio $=71 \%, \mathrm{ds}=5$, Hmáx $=76 \%$, Hmín $=67 \%, \mathrm{n}=6$, y Chatas: Temperatura promedio $=23,5^{\circ} \mathrm{C}$, ds $=0$, Tmáx $=23,8^{\circ} \mathrm{C}$, Tmín $=22,8^{\circ} \mathrm{C}, \mathrm{n}=6$; Humedad relativa promedio $=77 \%$,ds $=2$, HRmáx $=80 \%$, HRmín $=73 \%, n=6$ ). La precipitación varió con el tiempo y presentó una relación inversamente proporcional solo con la riqueza de aves del bosque Las Chatas ( $\mathrm{rs}=0,84, \mathrm{p}=0,07)$; la riqueza del bosque Colindres y su matriz y la abundancia en ambos sitios no presentó ninguna relación con la precipitación ( $\mathrm{p}>0,05)$.

La mayoría de las especies de los dos parches de bosque fueron catalogadas como raras $(77,55 \%$ Chatas y $70,59 \%$ Colindres) (Fig. 4). El número de especies por categoría de abundancia relativa entre ambos bosques fue similar $\left(\chi_{3 ; 0,05}^{2}=29,04\right.$; $\mathrm{p}=0,41)$. De las especies raras algunas fueron observadas sólo una vez como Amazilia saucerrottei, Ardea cocoi, Coccycua pumila, Myiarchus crinitus, Nyctibius griseus y Piaya cayana en las Chatas. Aramus guarauna, Ardea cocoi, Gymnomystax mexicanus, Machetornis rixosa, Ramphocelus dimidiatus, Saltator striatipectus y Sporophila nigricollis en Colindres. Las especies con mayor abundancia fueron 
Stelgydopterys ruficollis, Tyrannus melancholicus y Bubulcus ibis en el bosque de las Chatas y T. melancholicus en Colindres. En las matrices el número de especies por categoría de abundancia relativa fue diferente $\left(\chi_{3 ; 0,05}^{2}=8,85 ; \mathrm{p}=0,03\right)$; la matriz de Colindres presentó un número significativamente mayor de especies raras que la matriz de Las Chatas (Fig. 4).

Los índices de diversidad entre los bosques y entre las matrices, no presentaron diferencias significativas (Tabla 1). Sin embargo, al transformar cada índice de Shannon en "Número efectivo" el bosque Colindres y su matriz presentan un índice de diversidad mayor, siendo más diversos que Las Chatas. Tanto los bosques como las matrices presentan una comunidad con dominancia alta.
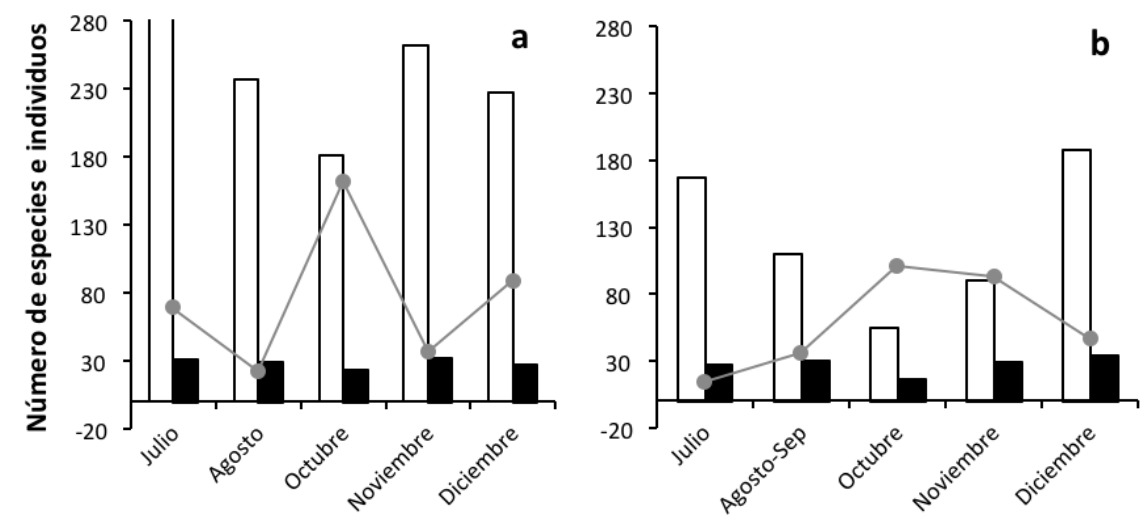

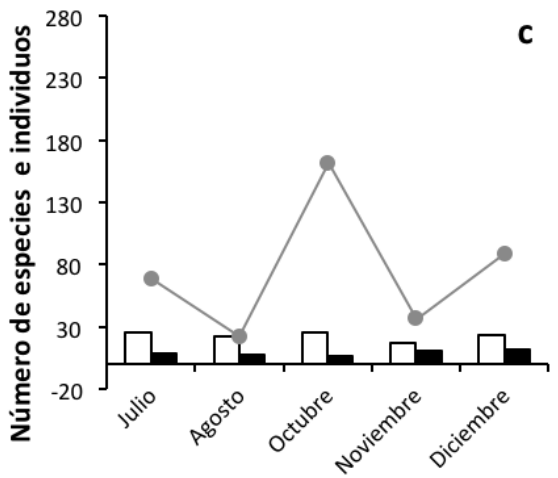

Meses muestreados

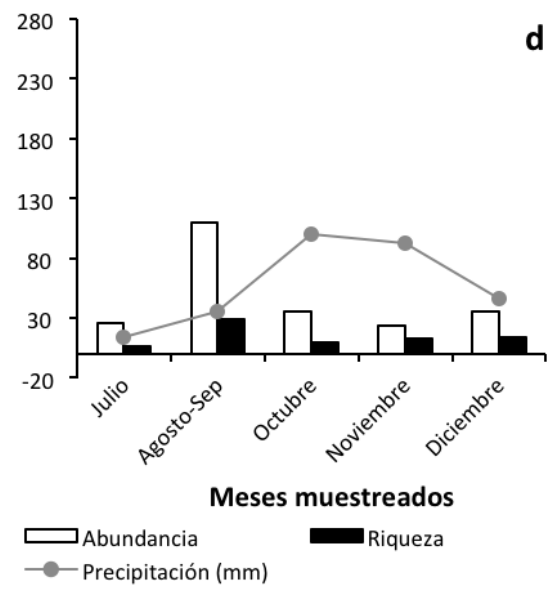

Figura 3. Variación temporal de la riqueza (número de especies) y abundancia (número de individuos) de aves y precipitación ( $\mathrm{mm}$ ) en los parches de bosque seco Las Chatas (a), Colindres (b), Matriz Las Chatas (c) y Matriz Colindres (d) en el Valle del Cauca, suroccidente de Colombia. 


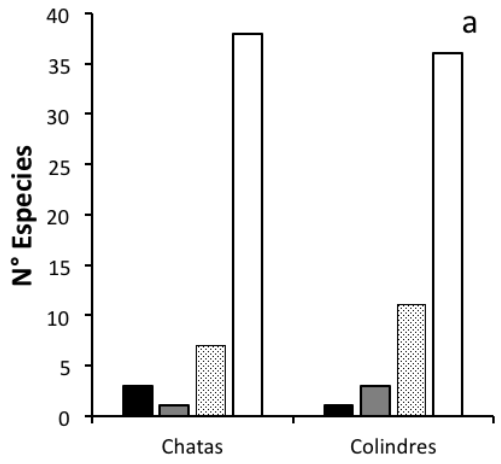

Categoría de abundancia

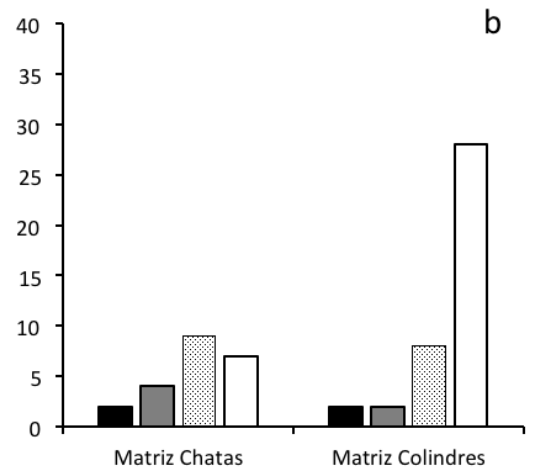

Categoría de abundancia

- Abundante $\square$ Común $\quad$ Poco común QRara

Figura 4. Número de especies por categoría de abundancia relativa en los parches de bosque seco Colindres y Las Chatas (a) y en sus respectivas matrices (b) en el Valle del Cauca, suroccidente de Colombia.

Tabla 1. Índices de diversidaden los parches de bosque seco Colindres y Las Chatas (a) y sus matrices (b) en el Valle del Cauca, suroccidente de Colombia.

\begin{tabular}{lllll}
\hline Sitio & Shannon & $\begin{array}{l}\text { Prueba de t para } \\
\text { Shannon }\end{array}$ & “Número efectivo" & Simpson 1-D \\
\hline Bosque Las Chatas & 2,95 & $\mathrm{t}_{(0,05)}=0,18 ; \mathrm{p}=0,86$ & 19,86 & 0,91 \\
Bosque Colindres & 3,36 & & 28,79 & 0,95 \\
Matriz Las Chatas & 2,79 & $\mathrm{t}_{(0,05)}=0 ; \mathrm{p}=1$ & 16,28 & 0,92 \\
Matriz Colindres & 2,99 & & 19,89 & 0,91 \\
\hline
\end{tabular}

La estructura trófica fue similar entre los bosques $\left(\chi^{2}{ }_{100,05}=32,27 ; p=0,98\right)$. En los dos parches dominan las especies consumidoras de insectos e invertebrados pequeños (IP), cuyas familias representativas fueron Tyrannidae, Picidae, Thamnophilidae, Parulidae, Furnariidae, Hirundinidae, Nyctibidae y Troglodytidae $(30,8 \%)$. El bosque Colindres presentó dos grupos exclusivos: los consumidores de frutas (F) (Fringillidae, Euphonia laniirostris) y los consumidores de insectos, invertebrados grandes y vertebrados muy pequeños (IV) (Accipitridae, Rosthramus sociabilis) (Fig. 5). La estructura trófica entre las matrices también fue similar $\left(\chi_{9 ; 0,05}^{2}=3,13 ; p=0,96\right)$, y las familias consumidoras de insectos más abundantes fueron: Tyrannidae, Hirundinidae, Picidae y Thamnophilidae (Fig. 5).

Con el análisis de agrupamiento de similitud en composición de especies de aves, Las Chatas y Colindres presentaron una similaridad del 46,49\%; mientras que, entre las matrices fué del 34,78\%.Ambos parches poseen un área similar $\chi_{1 ; 0.05}^{2}=0,34$; $\mathrm{p}=0,56$ ), difieren en la longitud del borde (perímetro: Colindres $1473 \mathrm{~m}$., Las Chatas 1220 m.) $\left(\chi_{1 ; 0,05}^{2}=23,8 ; p=0,001\right)$ y en su forma. En el bosque Colindres se 
encontraron 66 especies vegetales y en las Chatas 57, mientras en las matrices se encontraron 15 y 24 especies, respectivamente; la riqueza vegetal tanto en los parches de bosque como entre las matrices presentó una similaridad del 21,78\% y $14,7 \%$, respectivamente. Al evaluar si existía relación entre la riqueza de aves y la riqueza vegetal presente en los bosques y las matrices, se obtuvo que la correlación fue positiva y alcanzó un $80 \%$ ( $\mathrm{rs}=0,8 ; \mathrm{p}=0,083$ ). Las localidades ubicadas en condiciones similares de zona de vida más similares a estos dos parches de bosque, fueron El Hatico, Formosa y el CIAT con un 33\% de especies compartidas. Estas tres localidades constituyen un nodo que se separa en forma temprana en el análisis de agrupamiento del resto de las localidades (Fig. 6).
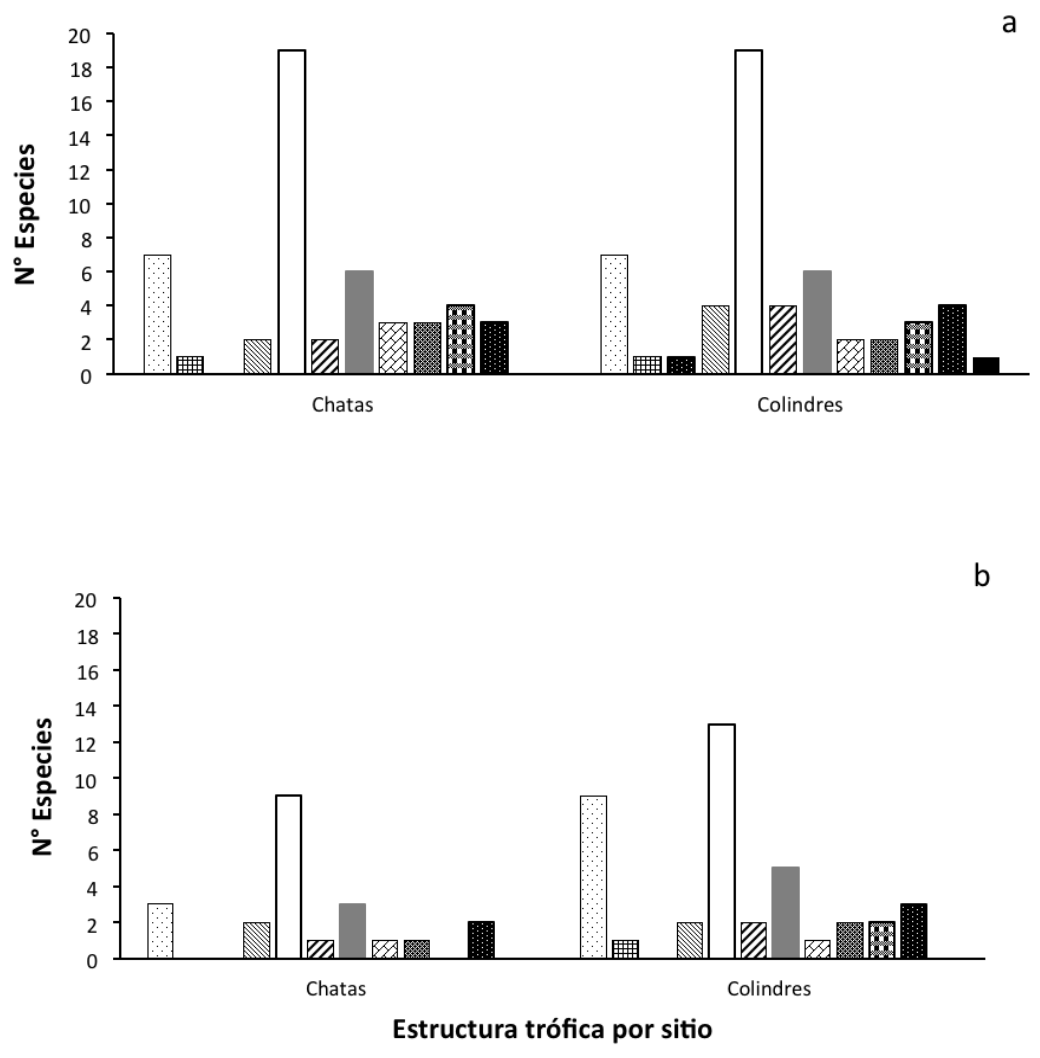

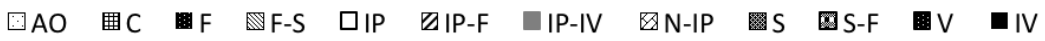

Figura 5. Grupos tróficos presentes en los parches de bosque seco Colindres y Las Chatas (a) y sus respectivas matrices (b), Valle del Cauca, suroccidente de Colombia. AO: acuático omnívoro, C: carroña, F: frutas, FS: frutas y semillas, IP: insectos e invertebrados pequeños, IPF: insectos, invertebrados pequeños y frutas, IPIV: insectos, invertebrados pequeños, invertebrados grandes y vertebrados muy pequeños, NIP: néctar, insectos e invertebrados pequeños, S: semillas, SF: semillas y frutas, V: vertebrados grandes y IV: invertebrados grandes y vertebrados muy pequeńos. 

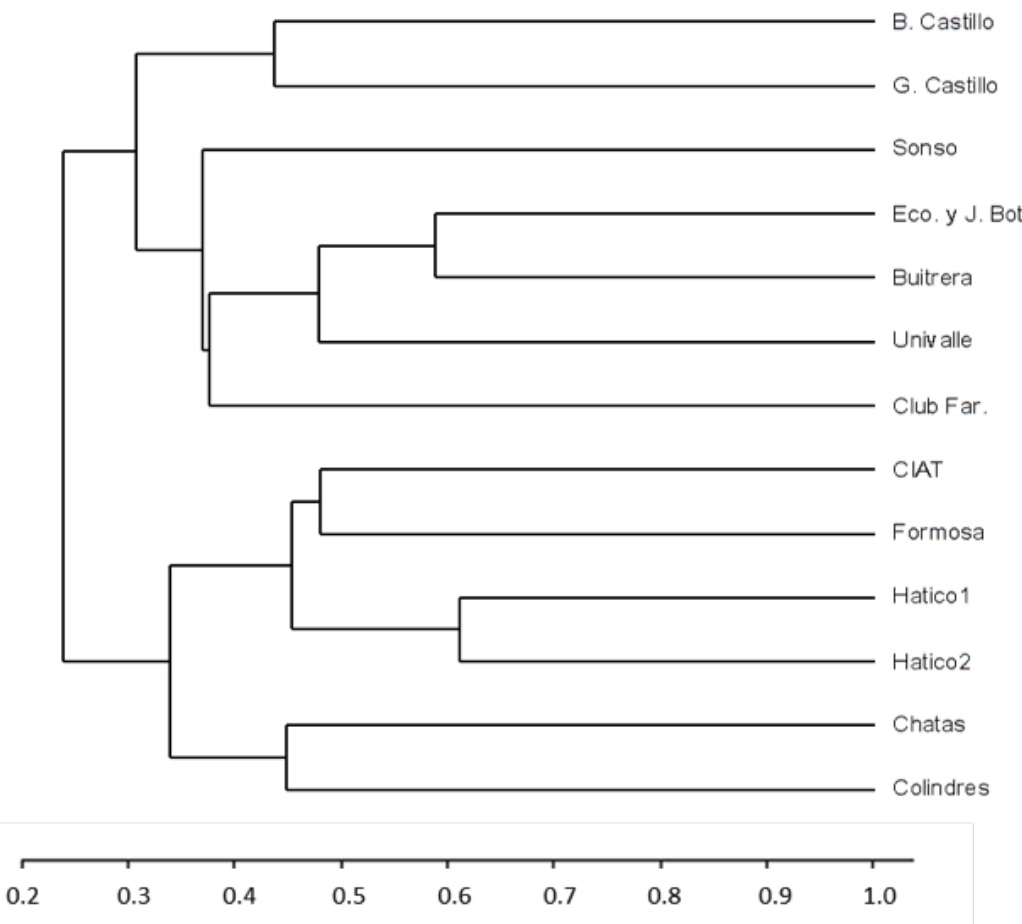

Similitud

Figura 6. Análisis de agrupamiento (índice de Jaccard) de los listados de especies de aves de Colindres, Chatas, CIAT: Centro Internacional de Agricultura Tropical, sede Palmira (Sedano, 2000), Hatico 1 y 2 (Cárdenas, 1998, Hurtado-Giraldo \& Cruz-Bernate datos no publicados, respectivamente), Bosque Hacienda el Castillo (Orejuela et al.1979), Guadual Hacienda el Castillo (Orejuela, 1979), Laguna de Sonso (Álvarez-López, 1999), Ecoparque del río Cali y Jardín Botánico (Fuentes, 2010), Univalle (Muñoz et al.2007), Buitrera (RiveraGutiérrez, 2006), Club Farallones de Cali (Naranjo \& Estela, 1999), Hacienda Formosa (Naranjo, 1992), en el Valle del Cauca, Colombia.

Los dos métodos de muestreo de observación (puntos de conteo y recorridos) mostraron un porcentaje significativo de especies compartidas, sobre todo para el parche boscoso Las Chatas (92,5\%); mientras que, en Colindres fue menor el número de especies compartidas (58,93\%).

\section{DISCUSIÓN}

La avifauna que presentan los parches de bosque seco Colindres y Las Chatas es similar en riqueza, diversidad, dominancia, estructura trófica y es típica de ambientes intervenidos que poseen amplia distribución geográfica (NARANJO, 1992; STOTZ et al. 1996; MARÍN-GÓMEZ, 2005, MURGUEITIO et al. 2008; FUENTES, 2010). La similitud en riqueza y número de especies por familia en los dos bosques, puede estar influenciada principalmente por la semejanza en área, ya que se conoce que 
sectores de vegetación del mismo tamaño conservan número de especies similares (FREEMARK \& MERRIAN, 1986; BERGET, 2006). Investigaciones en bosques secos en el Valle del Cauca, muestran también esta relación, donde zonas arbóreas con áreas entre 100 y 300 ha, como la finca ganadera Formosa, la reserva natural El Hatico, el Centro Internacional de Agricultura Tropical CIAT, las zonas verdes de la ciudad de Cali y el campus de la Universidad del Valle, albergan entre 90 y 140 especies de aves (NARANJO, 1992; CÁRDENAS, 1998; SEDANO, 2000; ANGARITA, 2002; MUÑOZ et al. 2007). Igualmente, la teoría de biogeografía de islas establece que hay relación positiva entre el número de especies y el área (MACARTHUR \& WILSON, 1967; KATTAN et al. 1994). En bosques templados en Canadá (Ottawa), un estudio de la diversidad de aves en 21 fragmentos de bosque de diferente tamaño, mostró que bosques más grandes y más heterogéneos en vegetación, tenían más especies que los bosques pequeños y la proporción de especies propias de interior de bosque, también aumentó (FREEMARK \& MERRIAN, 1986).

La diferencia en la riqueza encontrada entre las matrices, obedece probablemente a la calidad de la misma (KATTAN \& ÁLVAREZ-LÓPEZ, 1996; CÁRDENAS, 1998; PERFECTO et al. 2009). Según PERFECTO et al. (2009), la "calidad de la matriz" está relacionada con la cantidad de hábitat natural que ésta conserve y también de la gestión de los sistemas agrícolas cercanos; es decir, una matriz creada con técnicas agroecológicas, opuesta a la industria del monocultivo, aumentará los coeficientes de migración y otorgará recursos a diferentes especies. En la matriz del bosque Colindres se observaron especies acuáticas en el caño Potreritos, lo que indica que éste es un reservorio para aves acuáticas de la región. Aunque esta matriz presenta potreros y cultivo de caña de azúcar, también presenta un 21,8\% del borde donde hay numerosos árboles de manteco (Laetia americana) que son reconocidos hospederos de avifauna (TOKURA et al. 1996). También existen algunos parches de vegetación a los costados del caño, que sirven de refugio y favorecen la permanencia de varias especies y sus poblaciones (CALIDRIS, 2008).

La diferencia en número de individuos para cada bosque también puede verse influenciada, por la calidad de la matriz y el grado de intervención antrópica. Colindres y Las Chatas, remanentes de bosque inundable de mayor tamaño en el departamento del Valle del Cauca, perdieron esta condición debida a la canalización y como consecuencia de la ausencia de inundación, no existiendo el control natural sobre especies invasoras y oportunistas tanto faunísticas como florísticas, que aprovechan el nuevo hábitat disponible y degradado (ÁLVAREZ-LÓPEZ et al. 2009). Especies generalistas pertenecientes a las familias Tyrannidae, Emberizidae y Thraupidae también fueron encontradas en otros estudios en zonas intervenidas (NARANJO, 1992; CÁRDENAS, 1998; MARÍN-GÓMEZ, 2005; MURGUEITIO et al. 2008; VELÁSQUEZ-VALENCIA, 2009; FUENTES, 2010; MARÍN-GÓMEZ, 2012) y reportadas en los bosques Las Chatas y Colindres, aprovechan primeramente los bordes boscosos alterados y posteriormente penetran al interior del bosque (OREJUELA et al. 1979; FLORES et al. 2001; VELÁSQUEZ-VALENCIA, 2009; PORENSKY \& YOUNG, 2013). La caída de árboles robustos siempre dejan claros notables en el interior de los bosques y es allí donde se establecen estas especies (OREJUELA et al. 1979; FLORES et al. 2001, VELÁSQUEZ-VALENCIA, 2009; PORENSKY \& YOUNG, 2013). La presencia de ganado dentro de los parches, especialmente en Las Chatas, contribuye a que el suelo no tenga cobertura vegetal e imposibilita la recuperación gradual y sostenibilidad del mismo (SMITH et al. 2007; MURGUEITIO et al. 2008). Los efectos a corto plazo se relacionan con daños selectivos a plantas individuales de porte bajo 
y plántulas por herbivoría y pisoteo. A mediano y largo plazo ocurren cambios en las comunidades de plantas y animales, perturbaciones en el suelo y en los procesos hídricos, lo cual tiene consecuencias sobre la disponibilidad de recursos y hábitats para la biota nativa (MURGUEITIO et al. 2008). La pérdida de cobertura vegetal de porte bajo afecta a especies con preferencias de anidamiento en estratos y sustratos bajos (FUENTES, 2010), como especies de la familia Tinamidae, Caprimulgidae, Trochilidae, entre otras (STOTZ et al. 1996; MURGUEITIO et al. 2008). Otro factor que amenaza la diversidad de ambos bosques es la presencia de cazadores que extra en especies como Icterus nigrogularis, Dendrocygna bicolor, Anas discors y Ortalis columbiana (GIRALDOet al. 2007, Tamayo-Quintero Obs. pers).

Las especies más abundantes como Tyrannus melancholicus, Stelgidopteryx ruficollis, y Bubulcus ibis muestran un desbalance numérico característico de ambientes perturbados, empobrecidos o de ecosistemas jóvenes, donde existe una oferta mayor de recursos para este tipo de especies (CÁRDENAS, 1998; NARANJO \& ESTELA, 1999; VELÁZQUEZ-VALENCIA, 2009). Las Chatas presentó un gran número de claros dentro del bosque, lo cual según FITZPATRICK (1980) y SNOW (2004) favorece la presencia de especies que capturan su alimento en vuelo y usan perchas altas del dosel de bosque. En Las Chatas se evidenció la presencia de aves propias de zonas abiertas que presentan una abundancia y dominancia alta, similar a lo encontrado por FLORES et al. (2001). La presencia de claros trae consigo el crecimiento de especies vegetales de ciclos cortos y también fluctuaciones temporales de los artrópodos del follaje que son considerados factores importantes en los patrones de uso de los recursos por parte de aves insectívoras (BELTRÁN-SALAZAR, 2013).

El grupo trófico mayor representado fue el de los consumidores de insectos (30,8\% de las familias), al igual que lo encontrado en otros estudios de fragmentos de bosque intervenidos en la misma zona de vida (OREJUELA et al. 1979; NARANJO \& ESTELA, 1999; ANGARITA, 2002; VELÁZQUEZ-VALENCIA, 2009), y en agroecosistemas (NARANJO, 1992; SEDANO, 2000; CÁRDENAS et al. 2003; DE LA OSSA-LACAYO \& DE LA OSSA, 2014) y cuyas especies según VELÁSQUEZ-VALENCIA (2009), se ven favorecidas por la oferta alimenticia presente en los espacios abiertos. Especies consumidoras de frutas y/o semillas de las familias Thraupidae, Psittacidae, Fringillidae y los géneros Ortalis y Saltator son reconocidas por dispersar frutos y semillas (MOERMOND \& SLOAN, 1985), ayudando a mantener y restaurar la comunidad vegetal (PEJCHAR et al. 2008). La presencia de pocas especies de estos gremios en este estudio, puede indicar una baja tasa de reclutamiento asociado a la dispersión de semillas propias de plantas ornitócoras (VELASCO \& VARGAS, 2004). La forma y el mayor perímetro encontrado en el bosque Colindres contribuyen a que el área de interior de bosque sea reducida y por lo tanto se afecte la presencia de gremios especialistas, como lo encontrado en el bosque de San Antonio donde los frugívoros grandes y los insectívoros de sotobosque fueron los más afectados a la fragmentación del hábitat (KATTAN et al. 1994). A pesar de que las especies Piaya cayana, Nyctibius griseus, y Leptotila plumbeiceps en Las Chatas y Myiarchus apicalis en Colindres, se observaron sólo en el interior de cada bosque, estas especies no son consideradas exclusivas de interior de bosque (NARANJO, 1992; STOTZ et al. 1996; NARANJO \& ESTELA, 1999; MUÑOZ et al. 2007; MURGUEITIO et al. 2008; FUENTES, 2010).

Aunque Las Chatas y Colindres presentaron temperatura y humedad relativa parecidas durante la temporada de Julio a Diciembre de $2012\left(\chi_{4 ; 0,05}^{2}=0,03 ; p=1\right.$, 
$\chi_{4 ; 0,05}^{2}=0,82 ; \mathrm{p}=0,99$, respectivamente), la similitud en composición taxonómica avifaunística es relativamente baja, (45\%). La composición y la presencia de ciertos grupos tróficos como aquellas consumidoras de frutos y semillas, como Saltator striatipectus y Ortalis columbiana no fueron encontradas en el bosque Las Chatas, pero si en Colindres, indicando que al parecer existe una relación con la composición taxonómica vegetal interna, las zonas boscosas y la calidad de la matriz que rodean al bosque. Una mayor calidad de matriz (diversidad de hábitats naturales) está asociada con una mayor diversidad y mejores facilidades de desplazamiento (CÁRDENAS, 1998; ARMBRECHT \& ULLOA-CHACÓN, 1999; WETHERED \& LAWES, 2005; ROBLES \& CIUDAD, 2012; PORENSKY \& YOUNG, 2013). La mayor presencia de individuos de Laetia americana en la matriz del bosque Colindres, podría facilitar la migración de estas especies desde otras zonas boscosas a través del corredor de vegetación a lo largo de los ríos cercanos hasta el piedemonte del Parque Nacional Natural Farallones de Cali. Según NARANJO (1992), TOKURA et al. (1996), Fuentes (2010), HERNÁNDEZ et al. (2013) y CAICEDO-ARGUELLES \& CRUZ-BERNATE (2014), varias especies vegetales como Xylopialigus trifolia, Erythrina fusca, Pithecellobium sp., Inga ornata, Eugenia sp., Xilopia sp., Cecropia membranácea y Guazuma ulmifolia son fuente de alimento para la avifauna y se encuentran presentes en Colindres al igual que Acathocarpus nigricans, Senna reticulata, Pithecellobium sp., Guazuma ulmifolia y Eugenia oerstediana encontradas en el bosque Las Chatas. En las Chatas la presencia de especies vegetales diferentes a las de Colindres y árboles seniles favoreció la presencia de especies anidadoras en cavidades como Pionus menstruus y Forpus conspicillatus. A pesar de estas características, la movilidad de las especies a través de la matriz (RENJIFO, 2001; PERFECTO et al. 2009) probablemente está muy limitada porque todo el sector, incluyendo la Reserva Natural Laguna de Sonso que podría servir como fuente de especies para el bosque (KATTAN, 2002), se encuentra circunscrita en terrenos arados para monocultivos (CORPORACIÓN AUTÓNOMA REGIONAL DEL VALLE DEL CAUCA-CVC, 2014).

El análisis de agrupamiento con base en el índice de Jaccard, mostró que tanto Las Chatas como Colindres, están más relacionadas con El Hatico (CÁRDENAS, 1998, HURTADO-GIRALDO \& CRUZ-BERNATE, datos no publ.), la Hacienda Formosa (NARANJO, 1992) y el CIAT (SEDANO, 2000) con un 33\% de similaridad; éstos sitios comparten características como ser parches boscosos pequeños rodeados de diferentes sistemas de producción y reservorios de agua. Sin embargo, esta similaridad fue baja, probablemente debido a diferencias en los métodos de observación, intensidad del muestreo, y época en la que se realizó cada estudio. El trabajo realizado por CÁRDENAS (1998) empleó el método de puntos de conteo; mientras que, NARANJO (1992), SEDANO (2000) y HURTADO-GIRALDO \& CRUZBERNATE (datos no publ.) utilizaron un recorrido de avistamiento por rutas fijas. El esfuerzo de muestreo de estos estudios variaron entre siete meses y dos años, los sitios presentaron diferencias en cobertura arbórea, desde zonas arborizadas con reservorios de agua, cultivos y praderas artificiales (NARANJO, 1992; SEDANO, 2000), hasta zonas con sistemas de producción silvopastoril, cañaduzales, guaduales, bosque secundario y frutales (CÁRDENAS, 1998; HURTADO-GIRALDO \& CRUZ-BERNATE, datos no publ.).

En el bosque de Las Chatas la observación por puntos de conteo fue el método más eficaz para determinar la riqueza de especies, registrando 43 especies de un total de 49 observadas dentro del bosque. Por otro lado, en Colindres el método de observación por recorrido registró una mayor riqueza (45 especies de un total 
de 51). Esto indica que, en zonas con una estructura vegetal más compleja como Colindres, la observación por recorrido es el método que da una mejor aproximación a la avifauna del sitio. Por otra parte, las redes de niebla contribuyeron solo con tres especies diferentes a las observadas por medio de los puntos de conteo y observación por recorrido, por lo que este método, aunque es complementario, no es suficiente para evaluar la composición de especies de aves (STILES \& ROSELLI, 1998), ya que en las redes de niebla la población muestreada solo consiste de individuos de especies típicas del sotobosque.

La presencia de especies distintas entre los bosques, tales como Myiarchus apicalis y Picumnus granadensis, especies endémicas (HILTY \& BROWN, 2001) y algunas migratorias indican la importancia de la conservación de varios parches de vegetación en la misma zona de vida, puesto que pueden contribuir de manera complementaria con el mantenimiento de poblaciones de aves residentes y migratorias del bosque seco tropical.

\section{AGRADECIMIENTOS}

A la familia Villalobos y a la familia Calero propietarios del Bosque Las Chatas y Colindres, respectivamente. Al Departamento de Biología de la Universidad del Valle y a la Corporación Autónoma regional del Valle del Cauca (CVC) por su apoyo logístico y económico. Al Dr. William Beltrán y revisores por sus sugerencias al manuscrito.

\section{BIBLIOGRAFIA}

ÁLVAREZ-LÓPEZ, H., GIRALDO, M. AND KATTÁN, G., 1991.- Estado del conocimiento y la conservación de la Avifauna del Departamento del Valle del Cauca.Memorias Primer Simposio Nacional de Fauna del Valle del Cauca,INCIVA, pp. 335-353.

ÁLVAREZ-LÓPEZ, H., 1999.- Guía de las aves de la Reserva Natural Laguna de Sonso. Corporación Autónoma Regional del Valle del Cauca-CVC, Cali, pp. 107.

ÁLVAREZ-LÓPEZ, H., SALAZAR-RAMÍREZ, M. I., CARMONA-TOBAR, A. M., GÓMEZ-HOYOS, N., FLÓREZ, P. E., RIVERA, M. I., CALERO, A., SANDOVAL, M. C., MONDRAGÓN, C. E., VEGA-VARGAS, W. BOLÍVAR, W., CASTRO-HERRERA, F., ROJAS, V., REYES, M., QUINTERO, H. J., GONZÁLEZ E, C. A., BALANZA, C., ARANA, A. E., OREJUELA, J. E., PALTA, M. V., BAENA, L. M. AND MUÑOZ, L., 2009.- Humedales del valle geográfico del río Cauca, en el departamento del Valle del Cauca: Génesis, biodiversidad y conservación. Corporación Autónoma Regional del Valle del Cauca-CVC, Cali.

ANGARITA, I., 2002.-Composición y estructura de la avifauna de la Ciudad de Cali: Tesis, Universidad del Valle, Facultad de ciencias, Cali.

ARMBRECHT, I. AND ULLOA-CHACÓN, P., 1999.-Rareza y diversidad de hormigas en fragmentos de bosque seco colombianos y sus matrices. Biotropica, Vol. 31(4), pp. 646-653.

ASOCIACIÓN CALIDRIS, 2008.-Conservación de la avifauna asociada a humedales de la zona sur del valle geográfico del río Cauca, un ecosistema estratégico para la región. Informe final convenio 007 del 2007, Asociación Calidris y Corporación autónoma regional del Valle del Cauca-CVC, Cali.

BELTRÁN-SALAZAR, J. W., 2013.- Repartición de recursos alimenticios entre cinco especies de aves insectívoras en una arboleda seca subtropical,en KATTAN, G. ECHEVERRY-GALVIS, M. AND LÓPEZVICTORIA, M. (Eds.), Libro de resúmenes del IV Congreso Colombiano de Ornitología, Pontificia Universidad Javeriana, Cali, pp. 95.

BERGET, C., 2006.- Efecto del tamaño y de la cobertura vegetal de parques urbanos en la riqueza y diversidad de la avifauna de Bogotá, Colombia. Gestión y Ambiente, Vol. 9(2), pp. 45-60.

CAICEDO-ARGUELLES, A.P.,AND CRUZ-BERNATE, L., 2014.-Actividades diarias y uso de hábitat de la reinita amarilla (Setophaga perechia) y la piranga roja (Piranga rubra) en un área verde urbana de Cali, Colombia. Ornithol. Neotrop. 25: 247-260.

CÁRDENAS, G., 1998.-Comparación de la composición y estructura de la avifauna en diferentes sistemas de producción: Tesis, Universidad del Valle, Facultad de ciencias, Cali.

CÁRDENAS G., HARVEY, C. IBRAHIM, M. AND FINEGAN, B.,2003.-Diversidad y riqueza de aves en diferentes hábitats en un paisaje fragmentado en Cañas Costa Rica. Agroforestería de las Américas, Vol. 10, pp. 
78-85.

CÁRDENAS, M., LLANO, M., TAMAYO, J. AND GARZÓN, E., 2013.-Monitoreo de biodiversidad en Bosque seco Tropical: Las Chatas y Colindres y seguimiento de la especie Maytennus aff. corei. Informe final Convenio Departamento de Biología de la Universidad del Valle y Corporación Autónoma Regional del Valle del Cauca-CVC, Cali.

CÁRDENAS, G., VIDAL-ASTUDILLO, V., LÓPEZ, H., GIRALDO, C., RUÍZ, C., SAAVEDRA-RODRÍGUEZ, C., FRANCO, P. AND GUTIÉRREZ-CHACÓN, C., 2013.- Inventarios de Fauna y Flora en Relictos de Bosque en el Enclave Seco del Río Amaime, Valle del Cauca, disponible en: http://www.gbif.org/dataset/ fdbbb939-ee0b-4c6e-8eb3-15ed92177306, (accessed 16 Marzo 2014).

CASTILLO, L. S. AND GONZÁLEZ-A., M. (Eds.), 2007.-Avances en la implementación del Plan de Acción en Biodiversidad del Valle del Cauca. Agenda de investigación en biodiversidad y vertebrados amenazados. Corporación Autónoma Regional del Valle del Cauca-CVC, Cali.

CENTRO DE INVESTIGACIÓN DE LA CAÑA DE AZÚCAR DE COLOMBIA, CENICAÑA, 2014.-Red meteorológica automatizada de la estación de Bocas del Palo y Buga, Valle del Cauca.

CHAVES, M. E. AND ARANGO, N. (Eds.), 1998.-Informe nacional sobre el estado de la biodiversidad. Instituto de Investigación de Recursos Biológicos Alexander von Humboldt, Ministerio del Medio Ambiente y PNUMA,Bogotá, D.C.

COLWELL, R. K., 2005.-EstimateS: Statistical estimation of species richness and shared species from samples. Version 7, available at: http://purl.oclc.org/estimates (accessed Diciembre 2012).

CORPORACIÓN AUTÓNOMA REGIONAL DEL VALLE DEL CAUCA-CVC,1990.-Comparación de cobertura de bosques y humedales entre 1957 y 1986 con delimitación de las comunidades naturales críticas en el Valle geográfico del Río Cauca.Informe 90-7, Corporación Autónoma Regional del Valle del Cauca, CVC, Cali.

CORPORACIÓN AUTÓNOMA REGIONAL DEL VALLE DEL CAUCA-CVC,2014.-Fotografías digitales de la zona plana del Valle geográfico del Río Cauca, Corporación Autónoma Regional del Valle del Cauca, CVC, Cali.

DE LA OSSA-LACAYO, A. AND DE LA OSSA, V. A.,2014.- Diversidad de aves insectívoro-arbóreas en cercas vivas, Montes de María y Golfo de Morrosquillo, Colombia. Rev. Colombiana Cienc. Anim., Vol. 6(1), pp. 171-176.

ESPINAL, L. S., 1967.-Visión ecológica del Departamento del Valle del Cauca, Universidad del Valle, Cali.

FITZPATRICK, J. W., 1980.-Foraging behavior of Neotropical tyrant flycatchers. The Condor, Vol. 82, pp. 43-57.

FLORES, B., RUMIZ, D. I., FREDERICKSEN, T. S. AND FREDERICKSEN, N., 2001.-Uso de claros de aprovechamiento por las aves, de un bosque húmedo tropical Boliviano. Informe técnico Proyecto de Manejo Forestal Sostenible, Objetivo Estratégico de Medio Ambiente, USAID, Bolivia.

FREEMARK, K. E., MERRIAN, H. G., 1986.- Importance of area and habitat heterogeneity to bird assemblages in temperate forest fragments. Biological Conservation, Vol. 36(2), pp. 115-141.

FUENTES, G. E., 2010.-Caracterización de la avifauna del Jardín Botánico de Cali y Ecoparque Río Cali: Tesis, Universidad del Valle, Facultad de Ciencias, Cali.

GALLI, A. E., LECK, C. F. AND FORMAN, R. T.,1976.- Avian distribution patterns in forest islands of different sizes in central New Jersey.Auk, Vol. 93, pp. 356-64.

GARCÍA-OLIVA, F., CESAR, I., MORALES, P. AND MAASS, J. M., 1994.- Forest topasture conversion influences on soil organic carbon dynamics in a tropical deciduous forest. Oecologia, Vol. 99, pp. 392-396.

GIRALDO, A., BOLÍVAR, W., OSPINA, F., GARCÉS, M. AND ORTÍZ, C., 2007.-Aves y mamíferos de interés para la comunidad del Norte del Cauca y centro del Valle del Cauca. Grupo de investigación en ecología animal, Universidad del Valle, Cali, pp.1-23.

HAMMER, Ø., HARPER, D. A. T. AND RYAN, P., 2001.- PAST: paleontological statistics software package for education and data analysis.Paleontología electronica, Vol. 4(1), pp. 9.

HERNÁNDEZ, O. MONTOYA, P. AND CARDONA, V., 2013.- Uso de especies vegetales por la comunidad de aves en el campus de la Universidad del Valle, in KATTAN, G. ECHEVERRY-GALVIS, M. AND LÓPEZ-VICTORIA, M. (Eds.), Libro de resúmenes del IV Congreso Colombiano de Ornitología, Pontificia Universidad Javeriana, Cali, pp. 95.

HILTY, S. L. AND BROWN, W. L., 2001.-Guía de aves de Colombia.American Bird Conservancy-ABC, Colombia.

JANZEN, D. H., 1988.-Tropical dry forests: The most endangered major tropical ecosystem, in WILSON, E. O. AND PETERS, F. M. (Eds.), Biodiversity, National Academy Press, Washington, D.C., pp. 130-137. JOST, L., 2006.- Entropy and diversity. Oikos, Vol. 113, pp. 363-375.

KATTAN, G. H., ÁLVAREZ-LÓPEZ, H. AND GIRALDO, M., 1994.-Forest fragmentation and bird extinctions: San Antonio eighty years later.Conservation Biology, Vol. 8, pp. 138-146.

KATTAN, G. AND ÁLVAREZ-LÓPEZ, H., 1996.-Preservation and Management of Biodiversity in Fragmented in the Colombian Andes, en SHELHAS, J. AND GREENBERG, R. (Eds.), Forest patches in tropical landscapes. Island Press, Washington, DC, pp. 3-18.

KATTAN, G., 2002.-Fragmentación: patrones y mecanismos de extinción de especies, en GUARIGUATA, M. R. AND KATTAN, G. (Eds.), Ecología y conservación de Bosques Neotropicales. Editorial Tecnológica de Costa Rica, pp. 561-590.

KAUFFMAN, K., 2005.-Guía de campo de las Aves de Norteamérica.Hillstar Editions L. C. New York, pp. 392.

KRICHER, J., 2010.-Un compañero Neotropical, Una introducción a los animales, plantas y ecosistemas del trópico del nuevo mundo.American BirdingAssociation, Inc., UnitedStates of America, pp. 462. 
LOZANO, F. H., MENDOZA, J. E., VARGAS, A. M., RENJIFO, L. M., JIMÉNEZ, E., CAYCEDO, P. C., VARGAS, W., ARISTIZÁBAL, S. L. AND RAMÍREZ, D. P., 2009.-Oportunidades de conservación en el paisaje rural (Fase I),en LOZANO, F. H. (Ed.), Herramientas de manejo para la conservación de biodiversidad en paisajes rurales. Instituto de Investigación de Recursos Biológicos Alexander von Humboldt y Corporación Autónoma Regional de Cundinamarca-CAR, Bogotá, D. C., Colombia, pp. 238.

LUQUE, S.S., LATHROP, R.G. AND BOGNAR, J.A., 1994.- Temporal and spatial changes in an area of the New Jersey Pine Barrens landscape.Landscape Ecology, Vol. 9(4): pp 287-300.

MACARTHUR, R.H., AND WILSON, E.O., 1967.-The theory of island biogeography. Princeton University Press, Princeton.

MAGURRAN, A. E., 1988.-Ecological diversity and its measurement.Princeton UniversityPress, New Jersey, pp. 179.

MARÍN-GÓMEZ, O.H., 2005.-Avifauna del campus de la Universidad del Quíndio.Boletín SAO, Vol. 15(2), pp. $42-59$.

MARÍN-GÓMEZ, O.H., 2012.- Inventario de la avifauna de la Reserva Natural La Montaña del Ocaso, Quimbaya, Quindío.Revista de la Asociación Colombiana de Ciencias Biológicas, Vol. 24, pp. 129-142.

MOERMOND, T.C. AND SLOAN, J.,1985.-Neotropical Avian Frugivores, Patterns of Behavior, Morphology, and Nutrition, with consequences for fruit selection.NeotropicalOrnithology, OrnithologicalMonographs No. 36. 1041p.

MUÑOZ, C., FIERRO-CALDERÓN, K. AND RIVERA-GUTIERREZ, H., 2007.-Las aves del campus de la Universidad del Valle, una isla verde urbana en Cali, Colombia.Ornitología Colombiana, Vol. 5, pp. 5-20.

MURGUEITIO, E., CUARTAS, C. AND NARANJO, J. (Eds.), 2008.-Ganadería del futuro: Investigación para el desarrollo. Fundación CIPAV, Cali, pp. 490.

MURPHY, R. G. AND LUGO, A. E., 1986.-Ecology of tropical dry forest.AnnualReview of Ecology and Systematics, Vol. 17, pp. 67-88.

NARANJO, L. G.,1992.-Estructura de la avifauna en un área ganadera en el Valle del Cauca, Colombia. Caldasia, Vol. 17(1), pp. 55-66.

NARANJO, L. G. AND ESTELA, F., 1999.- Inventario de la avifauna de un área suburbana de la ciudad de Cali.Boletín SAO, Vol. 10, pp. $11-27$.

OREJUELA, J. E., RAITT, RJ., ÁLVAREZ-LÓPEZ, H., BENALCAZAR, C. AND SILVA DE BENALCÁZAR, F., 1979.Poblaciones de aves en un bosque relictual en el valle del Río Cauca, cerca a Jamundí, Valle, Colombia. Cespedesia, Vol. 8, pp. 29-42.

OREJUELA, J. E., 1979.- Estructura de la comunidad aviaria en un guadual de Bambusa guadua en el municipio de Jamundí, Valle, Colombia. Cespedesia, Vol. 8, pp. 43 -58.

ORIANS, G. H., 1969.-On the evolution of mating systems in birds and mammals. The American Naturalist, Vol. 108 (934), pp. 589-603.

PEJCHAR, L., PRINGLE, R. M., RANGANATHAN, J., ZOOC, J. R., DURAN, G., OVIEDO, F. AND DAILY, G. C., 2008.- Birds as agents of seed dispersal in a human-dominated landscape in southern Costa Rica. Biological Conservation, Vol.141 (2), pp.536-544.

PERFECTO, I. VANDERMEER, J. AND WRIGHT, A., 2009.-Nature's matrix: linking agriculture, conservation and food sovereignty. Earthscan London, Sterling, VA.

PETTINGIL, O. S. JR., 1969.-Ornithology in laboratory and field.Burguess, Mineapolis, Minnesota, USA.

PORENSKY, L. M. AND YOUNG, T. P., 2013.- Edge-effect interactions in fragmented and patchy landscapes. Conservation Biology, Vol. 27 (3), pp. 509-519.

QGIS DEVELOPMENT TEAM, 2014.- QGIS Geographic Information System, Open Source Geospatial Foundation Project. http://qgis.osgeo.org

QUESADA, M. AND STONER, K. E., 2003.- Threats to the conservation of tropical dry forest in Costa Rica, in FRANKIE, G. W., MATA, A. AND VINSON, S. B. (Eds.), Biodiversity Conservation in Costa Rica: Learning the lessons in the seasonal dry forest. University of California Press, Berkeley, California, pp. 266- 269.

RALPH, C., SAUER, J. AND DROEGE, S., 1995.-Monitoring Bird Populations by point counts. Pacific Southwest Research Station, Forest Service, US. Department of Agricultura, Albany, California.

REMSEN, J. V., CADENA JR. C. D., JARAMILLO, A., NORES M., PACHECO, J. F., PÉREZ-EMÁN, J., ROBBINS, M. B., STILES, F. G., STOTZ, D. F. AND ZIMMER K. J., 2014.-A classification of the bird species of South America, American Ornithologists' Union, disponible en: http://www.museum.lsu.edu/ Remsen/ SACCBaseline.html (accessed Julio 2014).

RENJIFO, L.M., 2001.- Effect of natural and anthropogenic landscape matrices on the abundance of subandean bird species. Ecological Applications, Vol. 11(1), pp. 1431.

RIVERA-GUTIÉRREZ H. F., 2006.-Composición y estructura de una comunidad de aves suburbana en el sur occidente de Colombia. OrnitologíaColombiana, Vol. 1(4), pp. 28-38.

ROBLES, H. AND CIUDAD, C., 2012.-Influence of habitat quality, population size, patch size, and connectivity on patch-occupancy dynamics of theMiddle Spotted Woodpecker. Conservation Biology, Vol. 26(2), pp. 284-293.

RUDNICKY, T. C. AND HUNTER, M. L., 1993.- Reversing the Fragmentation Perspective: Effects of clearcutsize on bird species richness in Maine. Ecological Applications, Vol. 3, pp. 357-366.

SALAZAR-RAMÍREZ, M. I., GÓMEZ-HOYOS, N., VARGAS-VARGAS, W. G. AND BOLÍVAR-GARCÍA, W. 2002.-Bosques secos y muy secos del departamento del Valle del Cauca, Colombia, Corporación Autónoma Regional del Valle del Cauca-CVC, pp. 72. 
SEDANO, R., 2000.- Aves del CIAT,en SEGOVIA, R. J., SEDANO, R., REINA, G., LÓPEZ, G. AND VAN SCHOONHOVEN, A. (Eds.), Árboles, arbustos y aves en el agroecosistema del CIAT.Centro Internacional de Agricultura Tropical-CIAT, Cali, No. 317, pp. 33-51.

SMITH, T. M., SMITH, R. L., SANJOSÉ, E., CRESPO, C., GARMENDIA, A., SAMO, E. AND MEZQUITA, F., 2007.Ecología.Pearson, sexta edición, España, pp. 682.

SNOW, D. W., 2004.-FamilyCotingidae (Cotingas), en DEL HOYO, J., ELLIOT, A. AND CHRISTIE, D. (Eds.) Handbook of the Birds of the World.BirdLife International \& Lynx Editions, Barcelona.

STILES, F.G. AND ROSSELLI, L.,1998.-Inventario de las aves de un bosque altoandino: comparación de dos métodos.Caldasia, 20: 29-43.

STOTZ, D.F., FITZPATRIC, J.W., PARKER, T.A. AND MOSKOVITS, D.K., 1996.-Neotropical Birds: Ecology and conservation. Conservation International and the Field Museum of Natural History, The University of Chicago Press, Chicago and London.

TOKURA, Y., BOTERO, L., RONDON, M. AND VILLANUEVA, G., 1996.- Especies forestales del Valle del Cauca. Corporación Autónoma Regional del Valle del Cauca-CVC, Cali, pp. 349.

VELASCO-LINARES, P. AND VARGAS-RÍOS, O., 2004.- Dinámica de la dispersión de plantas ornitócoras, reclutamiento y conectividad en fragmentos de bosque altoandino secundario (Reserva natural protectora, Cogua Cundinamarca).Acta Biológica Colombiana, Vol. 9, No. 2.

VELÁSQUEZ-VALENCIA, A., 2009.- Estructura de la comunidad de aves en sistemas de producción del piedemonte amazónico. Informe final convenio Universidad Nacional y Universidad de la Amazonía, Florencia, Caquetá, pp. 1-51.

VILLAREAL, H., ÁLVAREZ, M., CÓRDOBA, S., ESCOBAR, F., FAGUA, G., GAST, F., MENDOZA, H., OSPINA, M. AND UMAÑA A. M., 2004.-Manual de métodos para el desarrollo de inventarios de biodiversidad. Programa de Inventarios de Biodiversidad, Instituto de Investigación de Recursos Biológicos Alexander von Humboldt, Bogotá, Colombia, pp. 236.

WETHERED R. AND LAWES, M. J., 2005.-Nestedness of bird assemblages in fragmented afromontane forest: the effect of plantation forestry in the matrix.Biological Conservation, Vol. 123, pp. 125-137.

WILLIS, E. O., 1979.-The composition of avian communities in remanescent woodlots in southern Brazil. Papéis Avulsos de Zoologia, São Paulo, Vol. 33(1), pp. 1-25.

ZAR J., 1999.-BiostatisticalAnalysis.Prentince Hall, Cuarta edición, New Jersey, pp. 663. 
Anexo 1. Composición, grupos tróficos y estatus de especies de aves enlos parches de bosque seco Colindres y Las Chatas, Valle del Cauca, suroccidente de Colombia.

\begin{tabular}{|c|c|c|c|c|c|c|}
\hline Especies & Colindres & Chatas & $\begin{array}{l}\text { Matriz } \\
\text { Colindres }\end{array}$ & Matriz Chatas & $\begin{array}{l}\text { Grupos } \\
\text { tróficos }\end{array}$ & Estatus \\
\hline \multicolumn{7}{|l|}{ Anseriformes } \\
\hline \multicolumn{7}{|l|}{ Anatidae } \\
\hline Dendrocygna bicolor & & & $\mathrm{x}$ & & $\mathrm{AO}$ & $\mathrm{R}$ \\
\hline Anas discors & & & $\mathrm{x}$ & & $\mathrm{AO}$ & M \\
\hline \multicolumn{7}{|l|}{ Galliformes } \\
\hline \multicolumn{7}{|l|}{ Cracidae } \\
\hline Ortalis columbiana & $\mathrm{x}$ & & & & F-S & $\mathrm{R}$ \\
\hline \multicolumn{7}{|l|}{ Suliformes } \\
\hline \multicolumn{7}{|l|}{ Phalacrocoracidae } \\
\hline Phalacrocorax brasilanus & & & $\mathrm{x}$ & & $\mathrm{AO}$ & $\mathrm{R}$ \\
\hline \multicolumn{7}{|l|}{ Pelecaniformes } \\
\hline \multicolumn{7}{|l|}{ Ardeidae } \\
\hline Nycticorax nycticorax & $\mathrm{x}$ & $\mathrm{x}$ & $\mathrm{x}$ & $\mathrm{x}$ & $\mathrm{AO}$ & $\mathrm{R}$ \\
\hline Butorides striata & $\mathrm{x}$ & $\mathrm{x}$ & & & $\mathrm{AO}$ & $\mathrm{R}$ \\
\hline Bubulcus ibis & $\mathrm{x}$ & $\mathrm{x}$ & $\mathrm{x}$ & $\mathrm{x}$ & IP-IV & $\mathrm{R}$ \\
\hline Ardea alba & & $\mathrm{x}$ & & & $\mathrm{AO}$ & $\mathrm{R}$ \\
\hline Ardea cocoi & $\mathrm{x}$ & $\mathrm{x}$ & $\mathrm{x}$ & & $\mathrm{AO}$ & $\mathrm{R}$ \\
\hline \multicolumn{7}{|l|}{ Threskiornithidae } \\
\hline Phimosus infuscatus & $\mathrm{x}$ & $\mathrm{x}$ & $\mathrm{x}$ & $\mathrm{x}$ & $\mathrm{AO}$ & $\mathrm{R}$ \\
\hline
\end{tabular}

Cathartiformes

Cathartidae

Coragyps atratus

$\begin{array}{lll} & \mathrm{x} & \mathrm{x}\end{array}$

C $\quad \mathrm{R}$

Accipitriformes

Accipitridae

\begin{tabular}{|c|c|c|c|c|c|}
\hline Rostrhamus sociabilis & $\mathrm{x}$ & & & IV & $\mathrm{R}$ \\
\hline Rupornis magnirostris & $\mathrm{x}$ & $\mathrm{x}$ & $\mathrm{x}$ & $\mathrm{V}$ & $\mathrm{R}$ \\
\hline
\end{tabular}

Gruiformes

Aramidae

\begin{tabular}{|c|c|c|c|c|}
\hline Aramus guarauna & $\mathrm{x}$ & $\mathrm{x}$ & $\mathrm{AO}$ & $\mathrm{R}$ \\
\hline \multicolumn{5}{|l|}{ Rallidae } \\
\hline Aramides cajaneus & $\mathrm{x}$ & & $\mathrm{AO}$ & $\mathrm{R}$ \\
\hline Porphyrio martinica & & $\mathrm{x}$ & $\mathrm{AO}$ & $\mathrm{R}$ \\
\hline
\end{tabular}

Charadriiformes

Charadriidae

Vanellus chilensis

$\mathrm{x}$

$\mathrm{X}$

$\mathrm{x}$

$\mathrm{x}$

$\mathrm{AO} \quad \mathrm{R}$

Columbiformes

Columbidae

Patagioenas cayennensis

Zenaida auriculata

$\mathrm{x}$

$\mathrm{x}$

$\begin{array}{ll}\text { S-F } & \text { R } \\ \text { S-F } & \text { R } \\ \text { S-F } & \text { R } \\ \text { S-F } & \text { R }\end{array}$

Leptotila plumbeiceps

$\mathrm{X}$

$\mathrm{X}$

R

Columbina talpacoti

$\mathrm{x}$

$\mathrm{X}$

$\mathrm{x}$

Cuculiformes

Cuculidae

Coccycua pumila 


\begin{tabular}{|c|c|c|c|c|c|c|}
\hline Especies & Colindres & Chatas & $\begin{array}{l}\text { Matriz } \\
\text { Colindres }\end{array}$ & Matriz Chatas & $\begin{array}{l}\text { Grupos } \\
\text { tróficos }\end{array}$ & Estatus \\
\hline Piaya cayana & & $\mathrm{x}$ & & & IP-IV & $\mathrm{R}$ \\
\hline Crotophaga ani & $\mathrm{x}$ & $\mathrm{x}$ & $\mathrm{x}$ & $\mathrm{x}$ & IP-IV & $\mathrm{R}$ \\
\hline Crotophaga major & $\mathrm{x}$ & & $\mathrm{x}$ & & IP-IV & $\mathrm{R}$ \\
\hline \multicolumn{7}{|l|}{ Caprimulgiformes } \\
\hline \multicolumn{7}{|l|}{ Nyctibidae } \\
\hline Nyctibius griseus & & $\mathrm{x}$ & & & IP & $\mathrm{R}$ \\
\hline \multicolumn{7}{|l|}{ Apodiformes } \\
\hline \multicolumn{7}{|l|}{ Trochilidae } \\
\hline Anthracothorax nigricollis & & $\mathrm{x}$ & & & N-IP & $\mathrm{R}$ \\
\hline Amazilia tzacatl & $\mathrm{x}$ & $\mathrm{x}$ & $\mathrm{x}$ & $\mathrm{x}$ & N-IP & $\mathrm{R}$ \\
\hline Amazilia saucerrottei & $\mathrm{x}$ & $\mathrm{x}$ & & & IP & $\mathrm{R}$ \\
\hline \multicolumn{7}{|l|}{ Piciformes } \\
\hline \multicolumn{7}{|l|}{ Picidae } \\
\hline Picumnus granadensis & & $\mathrm{x}$ & & & IP & $\mathrm{E}$ \\
\hline Melanerpes rubricapillus & & $\mathrm{x}$ & & $\mathrm{x}$ & IP & $\mathrm{R}$ \\
\hline Colaptes punctigula & & $\mathrm{x}$ & & & IP & $\mathrm{R}$ \\
\hline Dryocopus lineatus & $\mathrm{x}$ & $\mathrm{x}$ & $\mathrm{x}$ & $\mathrm{x}$ & IP & $\mathrm{R}$ \\
\hline Campephilus melanoleucos & $\mathrm{x}$ & & & & IP & $\mathrm{R}$ \\
\hline
\end{tabular}

Falconiformes

Falconidae

\begin{tabular}{|c|c|c|c|c|c|c|}
\hline Milvago chimachima & $\mathrm{x}$ & $\mathrm{x}$ & $\mathrm{x}$ & $\mathrm{x}$ & $\mathrm{V}$ & $\mathrm{R}$ \\
\hline Caracara cheriway & $\mathrm{x}$ & & $\mathrm{x}$ & & $\mathrm{V}$ & $\mathrm{R}$ \\
\hline Falco sparverius & $\mathrm{x}$ & & $\mathrm{x}$ & & $\mathrm{V}$ & $\mathrm{R}$ \\
\hline Falco femoralis & & $\mathrm{x}$ & & & $\mathrm{V}$ & $\mathrm{R}$ \\
\hline
\end{tabular}

Psittaciformes

Psittacidae

\begin{tabular}{lllllll} 
Pionus menstruus & $\mathrm{x}$ & $\mathrm{x}$ & $\mathrm{x}$ & $\mathrm{x}$ & $\mathrm{F}-\mathrm{S}$ & $\mathrm{R}$ \\
Amazona amazonica & $\mathrm{x}$ & & $\mathrm{x}$ & & $\mathrm{F}-\mathrm{S}$ & $\mathrm{R}$ \\
Forpus conspicillatus & & $\mathrm{x}$ & & $\mathrm{x}$ & $\mathrm{F}-\mathrm{S}$ & $\mathrm{R}$ \\
\hline
\end{tabular}

Passeriformes

Thamnophilidae

Cercomacra nigricans

x $\mathrm{x}$

Furnariidae

Synallaxis albescens

Tyrannidae

$\mathrm{x}$

Elaenia flavogaster

Phaeomyias murina

$\mathrm{x}$

Poecilotriccus sylvia

Todirostrum cinereum

Tolmomyias sulphurescens

Pyrocephalus rubinus

Fluvicola pica

Machetornis rixosa

Myiozetetes cayanensis

Pitangus sulphuratus

Tyrannus melancholicus

$x$

$\mathrm{x}$

$\mathrm{x}$

$\mathrm{x}$

$\mathrm{X}$

$\mathrm{X}$

$\mathrm{x}$

$\mathrm{x}$

IP R

IP

R

$\mathrm{X}$

$\mathrm{R}$

$\begin{array}{ll}\text { IP } & \text { R } \\ \text { IP } & \text { R } \\ \text { IP } & \text { R } \\ \text { IP } & \text { R } \\ \text { IP } & \text { R } \\ \text { IP } & \text { R } \\ \text { IP } & \text { R } \\ \text { IP } & \text { R } \\ \text { IP } & \text { R } \\ \text { IP } & \text { R } \\ \text { IP } & \text { R }\end{array}$




\begin{tabular}{|c|c|c|c|c|c|c|}
\hline Especies & Colindres & Chatas & $\begin{array}{l}\text { Matriz } \\
\text { Colindres }\end{array}$ & Matriz Chatas & $\begin{array}{l}\text { Grupos } \\
\text { tróficos }\end{array}$ & Estatus \\
\hline Tyrannus savana & $\mathrm{x}$ & $\mathrm{x}$ & $\mathrm{x}$ & $\mathrm{x}$ & IP & $\mathrm{R}$ \\
\hline Myiarchus apicalis & $\mathrm{x}$ & & & & IP & $\mathrm{E}$ \\
\hline Myiarchus crinitus & & $\mathrm{x}$ & & & IP & $\mathrm{M}$ \\
\hline \multicolumn{7}{|l|}{ Hirundinidae } \\
\hline Stelgidopteryx ruficollis & $\mathrm{x}$ & $\mathrm{x}$ & $\mathrm{x}$ & $\mathrm{x}$ & IP & $\mathrm{R}$ \\
\hline Hirundo rustica & & $\mathrm{x}$ & & & IP & $\mathrm{M}$ \\
\hline \multicolumn{7}{|l|}{ Troglodytidae } \\
\hline Troglodytes aedon & $\mathrm{x}$ & $\mathrm{x}$ & & & IP & $\mathrm{R}$ \\
\hline \multicolumn{7}{|l|}{ Turdidae } \\
\hline Catharus ustulatus & & $\mathrm{x}$ & & & IP-IV & M \\
\hline Turdus ignobilis & $\mathrm{x}$ & & $\mathrm{x}$ & & IP-IV & $\mathrm{R}$ \\
\hline \multicolumn{7}{|l|}{ Thraupidae } \\
\hline Ramphocelus dimidiatus & $\mathrm{x}$ & & $\mathrm{x}$ & & IP-F & $\mathrm{R}$ \\
\hline Thraupis episcopus & $\mathrm{x}$ & $\mathrm{x}$ & $\mathrm{x}$ & $\mathrm{x}$ & IP-F & $\mathrm{R}$ \\
\hline Thraupis palmarum & $\mathrm{x}$ & & & & IP-F & $\mathrm{R}$ \\
\hline Tangara vitriolina & $\mathrm{x}$ & $\mathrm{x}$ & & & IP-F & $\mathrm{R}$ \\
\hline Sicalis flaveola & $\mathrm{x}$ & $\mathrm{x}$ & $\mathrm{x}$ & & $S$ & $\mathrm{R}$ \\
\hline Volatinia jacarina & & $\mathrm{x}$ & & $\mathrm{x}$ & S & $\mathrm{R}$ \\
\hline Sporophila nigricollis & $\mathrm{x}$ & $\mathrm{x}$ & $\mathrm{x}$ & & $S$ & $\mathrm{R}$ \\
\hline \multicolumn{7}{|l|}{ Incertaesedis } \\
\hline Saltator striatipectus & $\mathrm{x}$ & & & & F-S & $\mathrm{R}$ \\
\hline \multicolumn{7}{|l|}{ Parulidae } \\
\hline Geothlypis philadelphia & $\mathrm{x}$ & & & & IP & M \\
\hline Setophaga pitiayumi & $\mathrm{x}$ & $\mathrm{x}$ & & & IP & $\mathrm{R}$ \\
\hline Setophaga petechia & & $\mathrm{x}$ & & & IP & $\mathrm{M}$ \\
\hline \multicolumn{7}{|l|}{ Icteridae } \\
\hline Gymnomystax mexicanus & $\mathrm{x}$ & & & & IP-IV & $\mathrm{R}$ \\
\hline Icterus nigrogularis & $\mathrm{x}$ & $\mathrm{x}$ & $\mathrm{x}$ & $\mathrm{x}$ & IP-IV & $\mathrm{R}$ \\
\hline \multicolumn{7}{|l|}{ Fringillidae } \\
\hline Euphonia laniirostris & $\mathrm{x}$ & & & & $\mathrm{F}$ & $\mathrm{R}$ \\
\hline
\end{tabular}

Grupos tróficos: AO: acuático omnívoro, C: carroña, F: frutas, F-S: frutas y semillas, IP: insectos e invertebrados pequeños, IP-F: insectos, invertebrados pequeños y frutas, IP-IV: insectos, invertebrados pequeños, invertebrados grandes y vertebrados muy pequeños, N-IP: néctar, insectos e invertebrados pequeños, S: semillas, S-F: semillas y frutas, V: vertebrados grandes y IV: invertebrados grandes y vertebrados muy pequeños.

Estatus: R-Residente, M-Migratorio y E-Endémico. 